\title{
Motivations for food choices in Irish teens from the National Teens' Food Survey II
}

\author{
A.N. Daly ${ }^{1}$, E.J. O’Sullivan ${ }^{1}$, B.A. McNulty ${ }^{2}$, J. Walton ${ }^{3}$ and J.M. Kearney ${ }^{1}$ \\ ${ }^{1}$ School of Biological and Health Sciences, Technological University Dublin, Dublin, Ireland, \\ ${ }^{2}$ UCD Institute of Food and Health, University College Dublin, Dublin, Ireland and \\ ${ }^{3}$ Department of Biological Sciences, Munster Technological University, Cork, Ireland
}

This abstract was awarded the student prize.

During adolescence, teens start making their own food choices, and while health and nutrition are important, practical and social concerns also play an influential role ${ }^{(1)}$. The present study aims to determine factors that may influence the food choices of Irish teens, using data from the Food Choice Questionnaire (FCQ) ${ }^{(2)}$ in the National Teens' Food Consumption Survey II (NTFSII 2019/20, $\mathrm{n}=428, \mathrm{M} / \mathrm{F}: 212 / 216)^{(3)}$.

The FCQ contains 36 items and assesses the level of importance placed on different motives when making food choices ${ }^{(2)}$. Principal Component Analysis, reliability testing, Pearson correlations and independent samples t-test analyses were run to determine the key motivations for food choice and their association with age, sex, BMI, Physical Activity (PA), Screen Time (ST), weight perception, daily energy intake (kcal) and daily fat, protein and carbohydrate intakes (as\% $\%$ Total Energy).

Eight motivating factors were identified for Irish teens' food choices, in order of importance: Sensory Appeal (SA), Price \& Availability (P\&A), Health \& Natural Content (HNC), Familiarity, Ease of Preparation (EoP), Mood, Weight Control (WC), and Ethical Concerns (EC). Girls placed more importance than boys on HNC $(r=0.16)$, WC $(r=0.21)$, Mood $(r=0.21)$, and EC $(r=0.20$, all $P<0.001)$. Age was positively correlated with $\operatorname{EoP}(r=0.21, P<0.001)$ and $\operatorname{Mood}(r=0.15, P<0.01)$. WC was positively correlated with BMI $(r=0.22)$, weight perception $(r=0.26$, both $P<0.001)$, and protein intake $(r=0.14, P<0.01)$, and negatively correlated with energy intake $(r=-0.25, P<0.001)$. HNC was positively correlated with PA $(r=0.19)$ and protein intake $(r=0.22$, both $P<0.001)$, and negatively correlated with ST $(r=-0.15, P<0.01)$. Mood was positive correlated BMI $(r=0.14, P<0.01)$. Familiarity was positively correlated with ST $(r=0.16, P=0.001)$. EC was negatively correlated with energy intake $(r=-0.21, P<0.001)$. No associations existed between fat or carbohydrate intakes and any motivation.

Health and practical aspects to food choice are important for teens, but taste remains a key influence. However, food choice motivations vary between the sexes and by age, BMI status and weight perception, where girls were more motivated by health, weight control, mood and ethical concern than boys, and older teens were more influenced by mood and ease of preparation than younger teens.

Both those classified as overweight and those who perceived they were overweight were motivated more by weight control and mood for their food choices, whereas those who perceived their weight to be correct placed more importance on health and natural content. Those motivated by weight control had lower energy and higher protein intakes, and those motivated by the health and natural content of food had more health promoting behaviours with higher PA and lower ST, and higher protein intakes. Understanding the motivations of teens' food choice can help understand why they struggle to meet dietary recommendations and help to develop more effective health promotion messages.

\section{Acknowledgments}

The author would like to acknowledge funding from the Department of Agriculture, Food and the Marine, Ireland, and the IUNA network.

\section{References}

1. Fleming CA, Hookey K, Schmeid V et al. (2020) UNICEF Report.

2. Steptoe A, Pollard TM \& Wardle J (1995) Appetite 25, 267-284.

3. Irish Universities Nutrition Alliance (2021) NTFS II: Summary Report. [Available at: www.iuna.net]. 